\title{
EXTENSION D'UN THÉORÈME DE LA THÉORIE DES SÉRIES TRIGONOMÉTRIQUES.
}

PAR

\author{
G. CANTOR
}

a HALLE a. $\mathrm{s}$.

(Traduction d'un mém. publ. d. l. Annales math. de Leipsic t. V. p. 123.)

Je voudrais faire connaître dans ce travail une extension du théorème d'après lequel une fonction ne peut être développée que d'une seule manière en série trigonométrique.

J'ai cherché à démontrer dans le Journal de Crelle t. 72, p. 139, que deux séries trigonométriques:

$$
\frac{1}{2} b_{0}+\sum\left(a_{n} \sin n x+b_{n} \cos n x\right)
$$

et

$$
\frac{1}{2} b_{0}^{\prime}+\sum\left(a_{n}^{\prime} \sin n x+b_{n}^{\prime} \cos n x\right)
$$

qui, pour toutes les valeurs de $x$, convergent et. ont la même somme, ont les mêmes coefficients; j’ai ensuite montré, dans une notice relative à ce travail, que ce théorème reste vrai, si, pour un nombre fini de valeurs de $x$, on renonce soit à la convergence, soit à l'égalité des sommes des deux séries.

L'extension que j'ai en vue ici consiste en ce que pour un nombre infini de valeurs de $x$ dans l'intervalle $[0 \ldots \ldots(2 \pi)]$ on peut renoncer 
à la convergence ou à l'accord des sommes de séries, sans que le théorème cesse d'être vrai.

Mais dans ce but je suis obligé de commencer par des explications, ou plutôt par quelques simples indications destinées à mettre en lumière les diverses manières dont peuvent se comporter des grandeurs numériques en nombre fini ou infini; je suis amené par là à donner quelques définitions, afin de rendre aussi courte que possible l'exposition du théorème en question, dont la démonstration se trouve au $\$ 3$.

\section{$\S 1$.}

Les nombres rationnels servent de fondement pour arriver à la notion plus étendue d'une grandeur numérique; je les désignerai sous le nom de système $A$, en y comprenant zéro.

On rencontre une première généralisation de la notion de grandeur numérique dans le cas où l'on a, obtenue par une loi, une série infinie de nombres rationnels:

$$
a_{1}, a_{2}, \ldots \ldots a_{n}, \ldots \ldots
$$

constituée de telle sorte que la différence $a_{n+m}-a_{n}$ devient infiniment petite à mesure que $n$ croìt, quèl que soit le nombre entier positif $m$, ou, en d'autres termes, qu'avec $\varepsilon$ (positif rationnel) pris arbitrairement on a un nombre entier $n_{1}$ tel que $\left(a_{n+m}-a_{n}\right)<\varepsilon$, si $n \equiv n_{1}$, et si $m$ est un nombre entier positif pris à volonté.

J'exprime ainsi cette propriété de la série (1): „La série (1) a une limite déterminée $b$ ».

Ces mots ne servent donc qu'à énoncer cotte propriété de la série, sans exprimer d'abord autre chose, et de même que nous lions la série (1) avec un signe particulier $b$, de même on doit aussi attacher différents signes $b, b^{\prime}, b^{\prime \prime}$ à diverses séries de même espèce.

Soit une seconde série:

$$
a_{1}^{\prime}, a_{2}^{\prime}, \ldots a_{n}^{\prime}, \ldots
$$

Acta mathematica. 2. Imprimé 11 Juin 1883. 
ayant une limite déterminée $b^{\prime}$, on trouve que les deux séries (1) et ( $\left.1^{\prime}\right)$ ont constamment une des trois relations suivantes, qui s'excluent l'une l'autre: Ou bien: $1^{\circ} a_{n}-a_{n}^{\prime}$ devient infiniment petit $\grave{a}$. mesure que $n$ croìt, ou bien: $2^{\circ} a_{n}-a_{n}^{\prime}$, ̀̀ partir d'un certain $n$, reste toujours plus grand qu'une grandeur positive (rationnelle) $\varepsilon$, ou enfin $3^{\circ} a_{n}-a_{n}^{\prime}$, $\dot{a}$ partir d'un certain $n$ reste toujours plus petit qu'une grandeur négative (rationnelle) $-\varepsilon$.

Dans le cas de la première relation, je pose: $b=b^{\prime}$, dans le cas de la seconde: $b>b^{\prime}$, et, dans le cas de la troisième: $b<b^{\prime}$.

On trouve de même qu'une série (1), ayant une limite $b$, n'a avec un nombre rationnel $a$ qu'une des trois relations suivantes. Ou. bien:

$1^{\circ} a_{n}-a$ devient infiniment petit à mesure que $n$ augmente, ou bien: $2^{\circ} a_{n}-a$, ̀̀ partir d'un certain $n$, reste toujours plus grand qu'une grandeur positive (rationnelle) $\varepsilon$, ou enfin $3^{\circ} a_{n}-a$, à partir d'un certain $n$, reste toujours plus petit qu'une grandeur négative (rationnelle) $-\varepsilon$.

Pour exprimer l'existence de ces rapports, nous écrivons resp.:

$$
b=a, b>a, b<a .
$$

De ces définitions et de celles qui suivent immédiatement, il résulte (et on peut démontrer rigoureusement cette conséquence) que, $b$ étant la limite de la série (1), $b-a_{n}$ devient infiniment petit à mesure que $n$ croit, ce qui justifie par conséquent d'une manière précise la désignation de )limite de la série (1)) donnée à $b$.

Qu'on désigne par $B$ l'ensemble deş grandeurs numériques $b$.

D'après les conventions précédentes, on peut étendre les opérations élémentaires entreprises avec des nombres rationnels aux deux systèmes $A$ et $B$ réunis.

Soient en effet $b, b^{\prime}, b^{\prime \prime}$ trois grandeurs numériques du système $B$, les formules:

$$
b \pm b^{\prime}=b^{\prime \prime}, \quad b b^{\prime}=b^{\prime \prime}, \quad \frac{b}{b^{\prime}}=b^{\prime \prime}
$$

servent ’̀ exprimer qu'entre les séries correspondantes aux trois nombres $b, b^{\prime}, b^{\prime \prime}:$

$$
\begin{aligned}
& a_{1}, a_{2}, \ldots \\
& a_{1}^{\prime}, a_{2}^{\prime}, \ldots \\
& a_{1}^{\prime \prime}, a_{2}^{\prime \prime}, \ldots
\end{aligned}
$$


se vérifient resp. les relations:

$$
\begin{aligned}
& \lim \left(a_{n} \pm a_{n}^{\prime}-a_{n}^{\prime \prime}\right)=0 \\
& \lim \left(a_{n} a_{n}^{\prime}-a_{n}^{\prime \prime}\right)=0 \\
& \lim \left(\frac{a_{n}}{a_{n}^{\prime}}-a_{n}^{\prime \prime}\right)=0
\end{aligned}
$$

où je n'ai plus besoin, d'après ce qui précède, d'expliquer plus longuement le sens du signe-lim. On a des définitions semblables pour les cas où un ou deux des trois nombres appartiennent au système $A$.

En général toute équation obtenue par un nombre fini d'opérations élémentaires

$$
F\left(b, b^{\prime}, \ldots b^{(p)}\right)=0
$$

se présentera comme expression d'une relation déterminée entre les séries qui donnent naissance aux grandeurs numériques $b, b^{\prime}, b^{\prime \prime}, \ldots \ldots b^{(p)} \cdot\left(^{1}\right)$ Le système $A$ a donné naissance au système $B$; de même les deux systèmes $B$ et $A$ réunis, donneront naissance, par le même procédé, à un nouveau système $C$.

Soit en effet une série infinie:

$$
b_{1}, b_{2}, \ldots . b_{n}, \ldots \ldots
$$

de nombres choisis dans les systèmes $A$ et $B$ et n'appartenant pas tous au système $A$, et cette série étant constituée de telle sorte que $b_{n+m}-b_{n}$ devient infiniment petit à mesure que $n$ croit, quel que soit d'ailleurs $m$ (et cette condition, d'après les définitions précédentes, peut se concevoir comme quelque chose de parfaitement déterminé) je dirai que cette série a une limite déterminée $c$.

Les grandeurs numériques $c$ constituent le système $C$.

Les définitions de l'équivalence, de l'inégalité en plus ou en moins, et celles des opérations élémentaires soit entre les grandeurs $c$, soit entre

(1) Quand on dit, par exemple, qu'une équation de $\mu^{\text {ème }}$ degré à coefficients entiers: $f(x)=0$, a une racine réelle $\omega$, cela signifie qu'on a une série: $a_{1}, a_{2}, \ldots \ldots a_{n}, \ldots$. de la même nature que la série (1) ayant pour limite le signe $\omega$, et jouissant en outre de la propriété:

$$
\lim f\left(a_{n}\right)=0 .
$$


ces grandeurs elles-mêmes et celles des systèmes $B$ et $A$, sont analogues aux définitions données plus haut.

Tandis que les systèmes $B$ et $A$ sont tels qu'on peut égaler chacun des $a$ à un $b$, mais non pas chacun des $b$ à un $a$, on peut au contraire égaler non seulement chacun des $b$ à un $c$, mais aussi chacun des $c$ à un $b$.

Bien que par là les systèmes $B$ et $C$ puissent dans une certaine mesure être regardés comme identiques il est essentiel, dans la théorie que j'expose ici (et d'après laquelle la grandeur numérique, n'ayant d'abord en elle-même, en général, aucun objet, ne paraît que comme élément de théorèmes qui ont une certaine objectivité, de ce théorème, p. ex., que la grandeur numérique sert de limite à la série correspondante) il est essentiel, dis-je, de maintenir la distinction abstraite entre les deux systèmes $B$ et $C$; aussi bien l'équivalence de deux grandeurs numériques $b, b^{\prime}$ empruntées au système $B$ n'entraine pas leur identité, mais exprime seulement une relation déterminée entre les séries auxquelles elles se rapportent.

Le système $C$ et ceux qui le précèdent produisent d'une manière analogue un système $D$, ceux-ci à leur tour, un autre système $E$, et ainsi de suite; par $\lambda$ de ces opérations (en considérant l'opération par laquelle on a passé de $A$ à $B$ comme la première) on arrive à un système $L$ de grandeurs numériques.

$\mathrm{Si}$ on se rappelle la suite des définitions données pour l'équivalence et l'inégalité en plus ou en moins de ces différentes grandeurs numériques et pour les opérations élémentaires qui permettent de passer d'un système à l'autré, le même rapport aura lieu avec ceux qui précèdent, à l'exception de $A$, en sorte qu'on pourra toujours égaler une grandeur numérique $l$ à une grandeur numérique $k, i, \ldots \ldots c, b$, et réciproquement.

On peut ramener à la forme d'égalités de ce genre-les résultats de l'analyse (abstraction faite de quelques cas connus) bien que (je ne l'indique ici qu'en égard à ces exceptions) la notion de nombre, si développée qu'elle soit ici, porte en soi le principe d'une extension nécessaire en ellemême et absolument infinie.

Il semble légitime, étant donnée une grandeur numérique, dans le système $L$, de se servir de cette expression: C'est une grandeur numérique, une valeur, ou une limite, de $\lambda^{\text {j̀me }}$ espèce; d'où l'on voit que j'emploie en général les mots grandeur numérique, valeur et limite dans le même sens. 
Extension d'un théorème de la théorie des séries trigonométriques.

Une équation $F\left(l, l^{\prime}, \ldots l^{(p)}\right)=0$ formée de nombres $l, l^{\prime}, \ldots . . l^{(p)}$ au moyen d'un nombre fini d'opérations élémentaires apparaît précisément, dans la théorie en question, comme l'expression d'un rapport déterminé entre $p+1$ séries $\lambda$ fois infinies de nombres rationnels; ces séries sont produites par les séries simplement infinies qui définissent tout d'abord les grandeurs $l, l^{\prime}, \ldots \ldots l^{(p)}$; on les obtient en remplaçant, dans les premières, les éléments par -les séries qui les définissent, en traitant de même les séries ainsi obtenues, qui en général seront doublement infinies, et en continuant ce procédé jusqu'à ce qu'on n'ait plus devant soi que des nombres rationnels.

Dans une autre circonstance je reviendrai avec plus de détail sur tou's ces rapports. Ce n'est pas non plus ici le lieu d'expliquer comment les conventions et les opérations dont j'ai parlé dans ce $\S$ peuvent servir à l'analyse infinitésimale. Dans ce qui suit, en exposant le rapport des grandeurs numériques avec la géométrie de la ligne droite, je me bornerai presque exclusivement aux théorèmes nécessaires, d'où l'on peut, si je ne me trompe, déduire le reste au moyen d'une démonstration purement logique. J'indique pour le comparer aux $\S 1$ et $\S 2$, le $10^{\circ}$ livre des Eléments d'Euclide; qui peut servir de point de comparaison en cette matière.

\section{$\S 2$.}

Les points d'une ligne droite sont déterminés quand, en prenant pour base une unité de mesure, on indique leurs distances, abscisses, d'un point fixe 0 de la ligne droite par le signe + ou - , suivant que le point en question se trouve dans la partie (fixée d'avance) positive ou négative de la ligne à partir de 0 .

Si cette distance a avec l'unité de mesure un rapport rationnel, elle est exprimée par une grandeur numérique du système $A$; dans l'autre cas, si le point est connu par une construction, on peut toujours imaginer une série:

$$
a_{1}, a_{2}, a_{3}, \ldots \ldots a_{n}, \ldots
$$


réalisant les conditions énoncées dans le $\S 1$, et ayant avec la distance en question une relation telle que les points de la droite, auxquels se rapportent les distances $a_{1}, a_{2}, \ldots, a_{n}, \ldots \ldots$, se rapprochent à l'infini du point à déterminer, à mesure que $n$ augmente.

Ce que nous exprimons, en disant: La distance du point à déterminer au point 0 est égale à $b$, quand $b$ est la grandeur numérique correspondant à la série (1).

On démontre ensuite que les conditions d'équivalence et d'inégalité en plus ou en moins de distances connues concorde avec les conditions d'équivalence et d'inégalité en plus ou en moins (définies dans le $\$ 1$ ), des grandeurs numériques correspondantes, qui représentent ces distances.

Il suit maintenant sans difficulté que les grandeurs numériques des systèmes $C, D \ldots$ sont aussi capables de déterminer des distances connues. Mais pour achever de faire reporter le lien que nous observons entre les systèmes des grandeurs numériques définies dans le $\S 1$ et la géométrie de la ligne droite, il faut ajouter encore un axiôme dont voici le simple énoncé: A chaque grandeur numérique appartient aussi, réciproquement, un point déterminé de la droite, dont la coordonnée est égale $\grave{a}$ cette grandeur numiérique dans le sens exposé dans ce $\left.\S .{ }^{1}\right)$

J'appelle ce théorème un axiôme, par ce qu'il est dans sa nature de ne pouvoir être démontré d'une façon générale.

Ce théorème sert aussi à donner supplémentairement aux grandeurs numériques une certaine objectivité, dont elles sont, toutefois, complètement indépendantes.

D'après ce qui précède, je considère un point de la droite comme déterminé, quand sa distance de 0 , précédée du signe convenable, est donnée comme grandeur numérique, valeur ou limite de $\lambda^{\text {eme }}$ espèce.

$\left.{ }^{1}\right)$ A chaque grandeur numérique appartient un point déterminé, mais à chaque point se rapportent, comme coordonnées, dans le sens ei-dessus, une quantité innombrable de grandeurs numériques égales; car, comme on l'a déjà fait entendre plus haut, il suit, de fondements purement logiques, que des points distincts ne peuvent pas répondre à des grandeurs numériques égales, et qu'un seul et même point ne peut se rapporter à des grandeurs numériques inégales, comme coordonnées. 
Eixtension d'un théorème de la théorie des séries trigonométriques.

Entrons maintenant plus pleinement dans notre sujet et considérons les relations qui se présentent, étant données des grandeurs numériques en nombre fini ou infini.

D'après ce qui précède on pent considérer les différentes grandeurs numériques comme correspondant une à une avec les différents points d'une ligne droite. Pour plus de clarté, et sans que cela soit essentiel, nous nous servirons, dans la suite, de ce mode de représentation, et, quand nous parlerons de points, nous aurons toujours en vue les valeurs par lesquelles on les obtient.

Pour plus de brièveté, jappelle système de valeurs un nombre donné, fini oú infini, de grandeurs numériques, et système de points un nombre donné, fini ou infini, de points d'une droite. Ce qui sera dit dans la suite des systèmes de points, peut s'appliquer immédiatement, d'après ce qui a été dit, aux systèmes de valeurs.

Etant donné, dans un intervalle fini, un système de points, il y a lieu, en général, d'envisager un second système de points déduit du premier d'une certaine manière, puis un troisième déduit du deuxième de la même façon, etc.; il est nécessaire de les étudier tous si l'on veut concevoir la nature du premier.

Pour définir ces nouveaux systèmes de points, définissons d'abord la notion du: point-limite d'un système de points.

Par point-limite d'un système de points $P$, j'entends un point de la droite tel que dans son voisinage, il y ait un nombre infini de points du système $P$; il peut d'ailleurs se faire que le point-limite appartienne à ce système. Et j'appelle voisinage d'un point tout intervalle dans lequel ce point est contenu. D'après cela il est facile de démontrer qu'un système composé d'un nombre infini de points a toujours pour le moins. un point-limite. Nous appelons point isolé de. $P$ tout point qui, appartenant à $P$, n'est pas en même temps point-limite de $P$.

C'est dès lors la condition déterminée de tout point de la droite par rapport à un système donné $P$, d'être ou de ne pas êtrè un point-limite de ce système et on a, aussi défini en même temps que le système $P$, le système de ses points-limites, que je désigne par $P^{\prime}$ et que j'appelle le premier système dérivé de $P$.

Si le système $P^{\prime}$ n'est pas composé d'un nombre fini de points, on peut en déduire par le même procédé un autre systèmé $P^{\prime \prime}$, que j'appelle 
le second système dérivé de $P$. Par $\nu$ opérations analogues on arrive à la notion du $\nu^{\text {ème }}$ système $P^{(\nu)}$ dérivé de $P$.

Si par exemple le système $P$ est composé de tous les points de la droite dont les abscisses sont rationnelles et comprises entre 0 et 1 (qu'on y comprenne, ou non, les limites), le systẹme dérivé $P^{\prime}$ se composera de tous les points de l'intervalle $(0 \ldots \ldots 1)$, y compris les limites 0 et 1 . Les systèmes suivantes $P^{\prime \prime}, P^{\prime \prime \prime}, \ldots$ ne diffèrent pas de $P^{\prime}$. Ou bien, si la quantité $P$ est composée des points dont les abscisses sont respect. $1, \frac{1}{2}, \frac{1}{3}, \ldots . \frac{1}{n}, \ldots$, le système $P^{\prime}$ se composera du seul point 0 et ne donnera naissance lui-mếme, par déduction, à aucun'autre.

Il peut arriver, et c'est le cas qui nous intéresse seul ici, qu'après $\nu$ opérations le système $P^{(v)}$ se compose d'un nombre fini de points et par conséquent ne donne lui-même naissance, par déduction, à aucun autre système; dans ce cas nous appellerons le système primitif $P$ de la $\nu^{\text {ème }}$ espèce; et il suit de là que $P^{\prime}, P^{\prime \prime}, \ldots$ sont alors de la $\overline{\nu-1}^{\text {èm }}$, de la $\overline{\nu-2}^{\text {ime }} \ldots$ espèce.

Dans cette théorie, l'ensemble de tous les systèmes d'espèce déterminée est donc considéré comme un genre particulier dans l'ensemble de tous les systèmes de points imaginables, et les systèmes de points que nous avons appelés de $\nu^{\text {sme }}$ espèce forment une espèce particulière dans ce genre.

Un seul point offre déjà un : exemple d'un système de points de $\nu^{\text {sme }}$ espèce, si on donne son ałscisse comme grandeur numérique de $\nu^{\text {ème }}$ espèce, satisfaisant à certaines conditions faciles à établir. Si en effet on décompose alors cette grandeur numérique pour obtenir les termes $\left(\mathrm{de} \overline{\nu-1}^{\text {ème }}\right.$ espèce) de la série qui lui correspond, si on décompose ces membres euxmêmes pour arriver aux termes (de $\overline{\nu-2}$ ème espèce) qui les constituent, et ainsi de suite, on finit par obtenir un nombre infini de nombres rationnels; et, si on se représente le système de points correspondant à ces nombres, elle sera de $\nu^{\text {ème }}$ espèce. $\left({ }^{1}\right)$

$\left({ }^{1}\right)$ Je le relève expressement, que ce n'est pas toujours le cas. En général le système de points ainsi engendrée par une grandeur numérique de $\nu^{\mathrm{e} m e}$ espèce peut être d'une espèce inférieure ou supérieure à la $\nu^{\text {ème }}$ espèce ou même n’être d'aucune espèce déterminée. 
Théorème. Si une équation ayant la forme:

$$
0=C_{0}+C_{1}+\ldots+C_{u}+\ldots \ldots
$$

où $C_{0}=\frac{1}{2} d_{0} ; \quad C_{n}=c_{n} \sin n x+d_{n} \cos n x$, est satisfaite pour toutes les valeurs de $x$, à l'exception de celles qui correspondent aux points d'un système de points $P$ de $\nu^{\text {eme }}$ espèce donnée dans l'intervalle $[0 \ldots(2 \pi)]$, où $\nu$ désigne un nombre entier aussi grand que l'on veut, je dis qu'on aura:

$$
d_{0}=0, \quad c_{n}=d_{n}=0 \text {. }
$$

Démonstration. Dans cette démonstration, comme la suite le fera voir, en parlant de $P$ on a en vue non-seulement le système donnée de $\nu^{\text {ème }}$ espèce des points exceptionnels dans l'intervalle $[0 \ldots(2 \pi)]$, mais encore le système produit sur la ligne entière infinie par la répétition périodique.

Considérons maintenant la fonction:

$$
F(x)=C_{0} \frac{x x}{2}-C_{1}-\frac{C_{2}}{4}-\ldots-\frac{C_{n}}{n n}-\ldots
$$

Il résulte de la nature d'un système $P$ de $\nu^{\text {ème }}$ espèce $q u$ 'il doit $y$ avoir un intervalle $(\alpha \ldots \ldots \beta)$, ò̀ ne se trouve aucun point de ce système; pour toutes les valeurs de $x$ comprises dans cet intervalle on aura donc, à cause de la convergence de notre série (1) que nous avons supposée:

$$
\lim \left(c_{n} \sin n x+d_{n} \cos n x\right)=0,
$$

par conséquent, d'après un théorème connu (v. $4^{\mathrm{e}}$ vol. de Annales math. p. 139):

$$
\lim c_{n}=0, \quad \lim d_{n}=0 .
$$

La fonction $F$ jouit donc des propriétés suivantes (v. Rizmann, Sur le moyen de représenter une fonction par une série trigonométrique, $\$ 8$ ):

- $1^{\circ}$ Elle reste continue pour toutes ces valeurs de $x$.

$2^{\circ} \lim \frac{F(x+a)+F(x-a)-2 F(x)}{a \alpha}=0$, si $\lim \alpha=0$, pour toutes les valeurs de $x$, excepté celles qui correspondent aux points du système $P$. 
$3^{\circ}$ On a: $\lim \frac{F(x+\alpha)+F(x-\alpha)-2 F(x)}{\alpha}=0$, si lim $\alpha=0$, pour toutes les valeurs de $x$ sans exception.

Je vais montrer maintenant que $F(x)=c x+c^{\prime}$. Pour cela je considère d'abord un intervalle quelconque $(p \ldots \ldots q)$ où il n'y a qu'un nombre fini de points du système $P$; soient $x_{0}, x_{1}, \ldots x_{n}$ ces points écrits d'après leur ordre de succession.

Je dis que $F(x)$ est linéaire dans l'intervalle $(p \ldots \ldots q)$; car $F(x)$, à cause des propriétés $1^{\circ}$ et $2^{\circ}$, est fonction linéaire dans chacun des intervalles obtenus en divisant $(p \ldots \ldots q)$ par les points $x_{0}, x_{1}, \ldots \ldots x_{n}$; comme en effet il n'y a de points exceptionnels dans aucun de ces intervalles, les conclusions appliquées dans le mémoire (v. Journal de Borchardt, t. 72, p. 159) ont ici toute leur force; il ne reste donc à démontrer que l'identité de ces fonctions linéaires.

Je vais le faire pour deux fonctions voisines et je les choisis dans les deux intervalles $\left(x_{0} \ldots x_{1}\right)$ et $\left(x_{1} \ldots x_{2}\right)$.

Soit dans $\left(x_{0} \ldots \ldots x_{1}\right) \quad F(x)=k x+l$ et dans $\left(x_{1} \ldots x_{2}\right) \quad F(x)=k^{\prime} x+l^{\prime}$.

A cause de $1^{\circ}$ on a $F\left(x_{1}\right)=k x_{1}+l$; puis, pour des valeurs assez petites de $\alpha$ :

$$
\begin{aligned}
& F\left(x_{1}+a\right)=k^{\prime}\left(x_{1}+a\right)+l^{\prime} \\
& F\left(x_{1}-a\right)=k\left(x_{1}-a\right)+l .
\end{aligned}
$$

On a ainsi, à cause de $\mathbf{3}^{\circ}$ :

$$
\lim \frac{\left(k^{\prime}-k\right) x_{1}+l^{\prime}-l+a\left(k^{\prime}-k\right)}{\alpha}=0
$$

pour $\lim \alpha=0$, ce qui n'est possible que si:

$$
k=k^{\prime}, \quad l=l^{\prime} .
$$

En résumé nous pouvons énoncer le résultat suivant:

A) गSoit $(p \ldots \ldots q)$ un intervalle quelconque, où il n'y a qu'un nombre fini de points du système $P, F(x)$ sera linéaire dans cet intervalle.)

Je considère ensuite un intervalle quelconque $\left(p^{\prime} \ldots \ldots q^{\prime}\right)$ qui ne contient qu'un nombre fini de points $x_{0}^{\prime}, x_{1}^{\prime}, \ldots \ldots x_{n}^{\prime}$ du premier sys- 
tème dérivé $P^{\prime}$; - et je dis d'abord que dans chacun des intervalles partiels obtenus en divisant $(p \ldots \ldots q)$ par les points $x_{0}^{\prime}, x_{1}^{\prime}, \ldots, \ldots$, p. ex. dans $\left(x_{0}^{\prime} \ldots \ldots x_{1}^{\prime}\right)$, la fonction $F(x)$ est linéaire

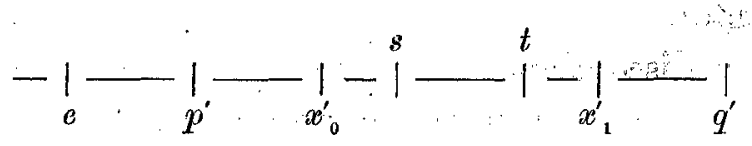

Car chacun de ces intervalles partiels contient il est vrai en général un nombre infini de points de $P$, en sorte que le résultat A) ne peut s'y appliquer immédiatement; mais chaque intervalle $(s \ldots \ldots t)$ compris dans les limites de $\left(x_{0}^{\prime} \ldots \ldots x_{1}^{\prime}\right)$ ne renferme qu'un, nombre fini de points de $P$. (parce qu'autrement il y aurait encore entre $x_{0}^{\prime}$ et $x_{1}^{\prime}$ d'autres points du système $\left.P^{\prime}\right)$ et par suite la fonction est linéaire dans $(s \ldots \ldots t)$ à cause de $\mathrm{A})$. Mais comme on peut rapprocher à volonté les points extrèmes $s$ et $t$ des points $x_{0}^{\prime}$ et $x^{\prime}{ }_{1}$, on conclut, sans façon que la fonction continue $F(x)$ est aussi linéaire dans $\left(x_{0}^{\prime} \ldots \ldots x_{1}^{\prime}\right)$.

Après l'avoir démontré pour chacun des intervalles partiels de $\left(p^{\prime} \ldots \ldots q^{\prime}\right)$, on obtient le résultat suivant par les mêmes raisonnements que ceux qui ont conduit au résultat $A$ ):

$\left.\mathrm{A}^{\prime}\right)$ Soit $\left(p^{\prime} \ldots \ldots q^{\prime}\right)$ un intervalle quelconque ne renfermant qu'un nombre fini de points du système $P^{\prime}, F(x)$ est linéaire dans cet intervalle.

La démonstration se poursuit de la même façon. Car, étant une fois établi que $F(x)$ est fonction linéaire dans un intervalle quelconque $\left(p^{(k)} \ldots \ldots q^{(k)}\right)$, qui ne contient qu'un nombre fini de points du $k^{\text {ème }}$ système $P^{(k)}$ dérivé de $P$, il résulte, comme dans le passage de $A$ ) à $\left.A^{\prime}\right)$, que $F(x)$ est aussi fonction linéaire dans un intervalle quelconque $\left(p^{(k+1)} \ldots \ldots q^{(k+1)}\right)$ qui ne renferme qu'un nombre fini de points du $(k+1)^{\text {ème }}$ système $P^{(k+1)}$.

Nous concluons ainsi, par un nombre fini de déductions successives, que $F(x)$ est linéaire dans tout intervalle qui ne contient qu'un nombre fini de points du système $P^{(\nu)}$. Mais le systëme $P$ étant de $\nu^{\text {ème }}$ espèce, comme on l'a supposé, un intervalle $(a \ldots \ldots b)$ pris à volonté dans la droite ne renfermera qu'un nombre fini de points de $P^{(\nu)}$. $F(x)$ est donc linéaire dans tout intervalle $(a \ldots \ldots b)$ pris à volonté, et il suit de là, comme il est facile de le voir, que $F(x)$ prend la forme: $F(x)=c x+c^{\prime}$ pour toutes les valeurs de $x$. Ce point étant mis en évidence, la démonstration se 
poursuit comme dans le travail déjà cité deux fois, à partir du moment où la forme linéaire est établie.

On peut aussi énoncer le théorème que nous avons démontré ici de la manière suivante:

》Une fonction discontinue $f(x)$, distincte de zéro ou indéterminée pour toutes les valeurs de $x$ correspondant aux points d'un système de points $P$ de $\nu^{\text {ème }}$ espèce donné dans l'intervalle $[0 \ldots(2 \pi)]$, mais égale à 0 pour toutes les autres valeurs de $x$, ne peut pas ètre représentée par une série trigonométrique.»

Halle, le 8 nov. 1871. 